\title{
Treatment and posttreatment effects of a facial mask combined with a bite-block appliance in Class III malocclusion
}

\author{
Paola Cozza, ${ }^{\text {a }}$ Tiziano Baccetti, ${ }^{b}$ Manuela Mucedero, ${ }^{\mathrm{c}}$ Chiara Pavoni, ${ }^{\mathrm{d}}$ and Lorenzo Franchi ${ }^{\mathrm{b}}$ \\ Rome and Florence, Italy
}

\begin{abstract}
Introduction: In this cephalometric investigation, we analyzed the treatment and posttreatment effects of an orthopedic protocol for Class III malocclusion consisting of a facial mask combined with a removable biteblock appliance. Methods: The treated sample consisted of 22 Class III patients treated with the facial mask and bite-block protocol before the pubertal growth spurt (mean age, $8.9 \pm 1.5$ years). Treated subjects were evaluated after facial mask and bite-block therapy and at a posttreatment observation in absence of retention. The treated group was compared with a matched control group of 12 untreated Class III subjects. All treated and control subjects were postpubertal at the final observation. Significant differences between the treated and control groups were assessed with the Mann-Whitney $U$ test $(P<0.05)$. Results: Both angular and linear sagittal measurements of the maxilla showed significant improvements during active treatment. Significant improvements of SNA angle, ANB angle, overjet, and molar relationship remained stable during the posttreatment period. No significant effect was found in the mandibular skeletal measures. No significant protraction of the maxillary incisors or retraction of the mandibular incisors was observed. Conclusions: A bite-block appliance in the mandibular arch with a facial mask enabled effective control of mandibular rotation with progressive closure of the gonial angle. This added to the favorable maxillary outcomes of the treatment protocol. (Am J Orthod Dentofacial Orthop 2010;138:300-10)
\end{abstract}

$\mathbf{T}$ reatment of Class III malocclusion in growing subjects is a challenging part of contemporary orthodontic practice. Many treatment approaches can be found in the literature regarding orthopedic and orthodontic treatment in Class III malocclusion, inlcuding intraoral and extraoral appliances such as facial mask (FM), ${ }^{1}$ FR-3 appliance of Fränkel, ${ }^{2}$ bionator, ${ }^{3}$ removable mandibular retractor, ${ }^{4}$ double-piece corrector, ${ }^{5}$ chincup, ${ }^{6}$ splints, Class III elastics, and chincup (SEC III), ${ }^{7}$ and mandibular cervical headgear. ${ }^{8,9}$

Initial observations on the effects of posteroanterior traction of the maxillary complex in the treatment of Class III malocclusion demonstrated skeletal protraction of the maxilla and the dentition, improvement of

aProfessor and head, Department of Orthodontics, University of Rome "Tor Vergata," Rome, Italy.

bAssistant professor, Department of Orthodontics, University of Florence, Florence, Italy; Thomas M. Graber Visiting Scholar, Department of Orthodontics and Pediatric Dentistry, School of Dentistry, University of Michigan, Ann Arbor.

cLecturer, Department of Orthodontics, University of Rome "Tor Vergata," Rome, Italy.

${ }^{\mathrm{d}}$ Research fellow, Department of Orthodontics, University of Rome "Tor Vergata," Rome, Italy

Reprint requests to: Lorenzo Franchi, Università degli Studi di Firenze, Via del Ponte di Mezzo, 46-48, 50127, Firenze, Italy; e-mail, 1.franchi@odonto.unifi.it. Submitted, May 2008; revised and accepted, September 2008. $0889-5406 / \$ 36.00$

Copyright (C) 2010 by the American Association of Orthodontists. doi:10.1016/j.ajodo.2010.05.001 the ANB angle, backward and downward rotation of mandible with augmentation of facial height, and lingual tipping of the mandibular incisors. ${ }^{10-13}$ These results were often confirmed in controlled trials that usually included combined maxillary expansion and FM therapy. ${ }^{14-21}$ Clinical studies have also shown variable effects of maxillary protraction therapy on the amount of mandibular growth, with a tendency toward Class III relapse when the mandible rotated downward and backward during treatment. ${ }^{17-19,21}$ Only a few studies dealing with the effects of orthopedic therapy of Class III malocclusion included posttreatment observations to evaluate relapse tendencies after active therapy. ${ }^{15,17-25}$ Also, few trials incorporated an untreated control sample of Class III subjects followed longitudinally. ${ }^{16}$

Predictive variables for satisfactory long-term outcomes of Class III early treatment can be identified in the overcorrection of overjet $(\mathrm{OJ})^{15,18}$ and the appropriate control of vertical dentoalveolar height to prevent an unfavorable clockwise rotation of the mandible. $^{19-21}$ Bite-blocks (BB) have demonstrated beneficial therapeutic effects in subjects with dentoskeletal open-bite tendency, since they reduce extrusion of the posterior teeth and allow autorotation of the mandible to produce bite closure. ${ }^{26}$ Extending these concepts, McNamara, ${ }^{11}$ Baccetti et al, ${ }^{14,24}$ and Westwood et al $^{15}$ 
used bonded maxillary expanders with acrylic splints as a BB device combined with FM therapy for Class III malocclusion. Favorable outcomes of BB therapy for the control of mandibular growth direction were observed in both experimental animal and human studies. $^{27-32}$

The purpose of this controlled clinical trial was to evaluate the therapeutic effects of a treatment protocol for Class III malocclusion consisting of a FM and a BB appliance in the mandibular arch. Both active treatment and posttreatment effects were analyzed in consecutively treated patients, and they were compared with the longitudinal growth changes in a matched control group of untreated Class III subjects.

\section{MATERIAL AND METHODS}

The treated group comprised 22 patients with dentoskeletal Class III malocclusion treated with a FM combined with a BB appliance it the mandibular arch, who were consecutively treated in the Department of Orthodontics at the University of Rome "Tor Vergata" in Italy. The success of therapy at the end of the observation period was not a determining factor for the selection of patients, since the treated sample was collected prospectively.

At the initial observation (T1), all patients had Class III malocclusion in the mixed dentition characterized by a Wits appraisal of $-2 \mathrm{~mm}$ or less, anterior crossbite or incisor end-to-end relationship, and Class III molar relationship. ${ }^{33}$ All patients were white, with a prepubertal stage of skeletal maturity according to the cervical vertebral maturation method (CS 1 or CS 2). ${ }^{34}$ No permanent teeth were congenitally missing or extracted before or during treatment. These Class III patients had no transverse discrepancy between the dental arches.

Lateral cephalograms were taken at $\mathrm{T} 1$ and the end of active treatment with the FM-BB protocol (T2). After active therapy, they were recalled every 3 months to assess the stability of treatment outcomes. The treated group was reevaluated at a follow-up observation (T3) with a third set of lateral cephalograms at an average time after $\mathrm{T} 2$ of about 2 years (about 3.5 years after T1). All subjects had reached postpubertal skeletal maturity at T3 (CS 4, CS 5, or CS 6). The stages of cervical vertebral maturation were determined by an operator (M.M.) trained in this method. All subjects had permanent dentitions at T3, when fixed appliance therapy could be used to refine the occlusion.

A control group of 12 untreated subjects with dentoskeletal Class III malocclusion was obtained from the Department of Orthodontics at the University of Florence in Italy. The control group matched the treated
Table I. Ages (y) of the Class III study groups and lengths of treatment or observation

\begin{tabular}{|c|c|c|c|c|}
\hline & \multicolumn{2}{|c|}{$\begin{array}{c}\text { Treated group } \\
(n=22 ; 9 \text { boys, } 13 \text { girls })\end{array}$} & \multicolumn{2}{|c|}{$\begin{array}{c}\text { Control group } \\
(n=12 ; 7 \text { boys, } 5 \text { girls })\end{array}$} \\
\hline & Mean & $S D$ & Mean & $S D$ \\
\hline $\mathrm{T} 1$ & 8.9 & 1.5 & 7.6 & 1.4 \\
\hline $\mathrm{T} 2$ & 10.5 & 1.3 & 9.8 & 1.9 \\
\hline T3 & 12.6 & 1.9 & 11.9 & 2.2 \\
\hline $\mathrm{T} 2-\mathrm{T} 1$ & 1.6 & 0.8 & 2.2 & 1.3 \\
\hline T3-T2 & 2.1 & 1.3 & 2.0 & 1.7 \\
\hline $\mathrm{T} 3-\mathrm{T} 1$ & 3.7 & 1.3 & 4.3 & 2.0 \\
\hline
\end{tabular}

group as to type of dentoskeletal disharmony, skeletal maturation at the various times, and duration of observation intervals. The mean ages at the time periods in both groups and the durations of either treatment or observation are given in Table I.

This study, therefore, comprised a treated sample that was collected prospectively and a control sample that was collected retrospectively.

The intraoral part of the maxillary appliance (intraoral anchorage appliance for posteroanterior elastics connecting with the FM) was constructed with a 1-mm stainless steel arch (buccal and palatal), with 2 hooks in the maxillary canine region to attach the elastics. The intraoral appliance was soldered to bands placed on the maxillary first permanent molars, and the palatal arch was placed in contact with the gingival margins of the maxillary teeth. Maxillary protraction began with forward and downward traction directed approximately $30^{\circ}$ to $40^{\circ}$ to the occlusal plane. Extraoral elastics were attached from the hooks on the intraoral appliance to the adjustable crossbar of the FM, so that the elastics did not interfere with the function of the lips. Hooks were soldered in the frontal part of the maxillary arch, between the lateral incisors and the deciduous canines, to obtain a direct effect of forward displacement of the maxilla. Elastics of increasing force were used during the first month of therapy until a heavy orthopedic force ( $600 \mathrm{~g}$ for each side) was delivered during the next treatment period. A Delaire FM was used to provide the extraoral anchorage. The patients were instructed to wear the FM at least 14 hours a day; cooperation was good for all of them.

During FM treatment, a BB appliance was used in all treated patients, with the aim to counteract any tendency to clockwise mandibular rotation. The BB appliance was constructed in the form of a Schwarz plate for the mandibular arch with a vestibular arch, occlusal resin splints, and an expansion screw that was activated when needed (Figs 1-3). The splints were intended to control molar eruption, limit 


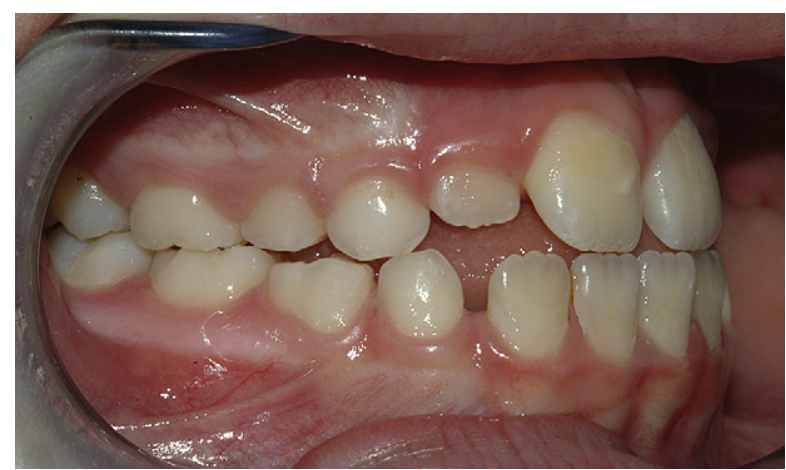

Fig 1. Boy (age, 8 years 7 months) with Class III malocclusion before treatment.

intermaxillary divergency, and prevent clockwise mandibular rotation. The patients were instructed to wear the BB for 24 hours a day, including during meals; cooperation was good for all of them.

All patients were treated at least to a positive dental OJ before discontinuing treatment; most patients were overcorrected toward a Class II occlusal relationship. No appliance was worn during the $\mathrm{T} 2$ to $\mathrm{T} 3$ posttreatment observation interval.

A customized digitation regimen and analysis were used for all cephalograms examined in this study. The regimen contained measurements from the analyses of Jacobson, ${ }^{33}$ McNamara, ${ }^{35}$ Ricketts, ${ }^{36}$ Steiner, ${ }^{37}$ and Tweed. $^{38}$

Before the cephalometric analysis, the intraobserver measurement error was evaluated. Fifteen lateral cephalograms, selected from various subjects in the study, were traced and measured at 2 times within a week by the same operator (M.M.). The measurements at both times for each patient were analyzed with the intraclass correlation coefficient, which varied between 0.966 for the SNB angle and 0.995 for the inclination of the maxillary incisor to the Frankfort horizontal (FH) line. These values indicated a high level of intraobserver agreement. Linear measurement errors averaged 0.3 $\mathrm{mm}(\mathrm{SD}, 0.8 \mathrm{~mm})$, and angular measurement errors averaged $0.4^{\circ}\left(\mathrm{SD}, 0.6^{\circ}\right)$.

Each cephalogram was traced at the 3 times, and 29 variables (14 linear, 15 angular) were measured. The cephalometric measurements used were the following (Figs 4 and 5): (1) cranial flexure, NSBa angle; (2) maxillary skeletal, SNA angle, A-N perpendicular (Point A to a line drawn perpendicular to the FH from N), CoANS, and Co-A; (3) mandibular skeletal: SNB angle, $\mathrm{Pg}-\mathrm{N}$ perpendicular (Pg to a line drawn perpendicular to the FH from N), Co-Go, Ar-Go, Co-Gn, and GoGn; (4) skeletal differences: ANB angle, Wits appraisal (distance between the 2 points of intersection

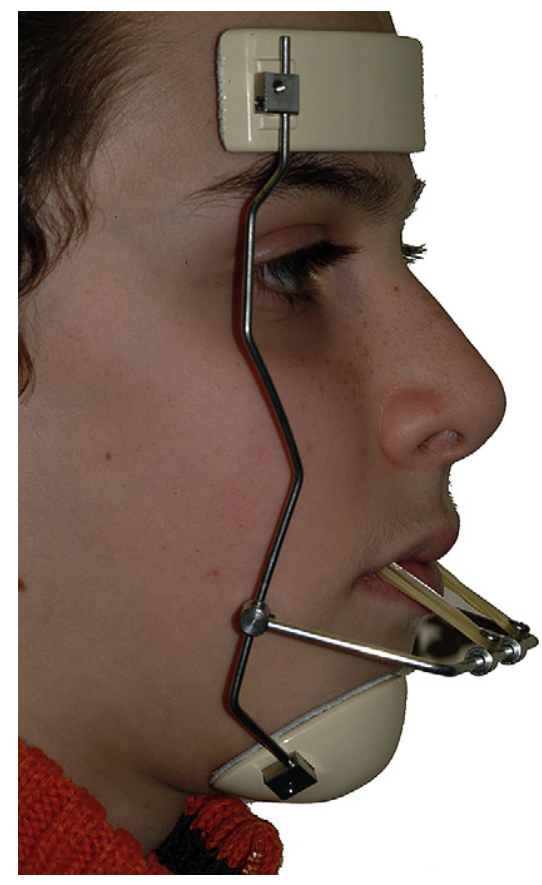

Fig 2. Facial mask: note the downward direction of the extraoral elastics.

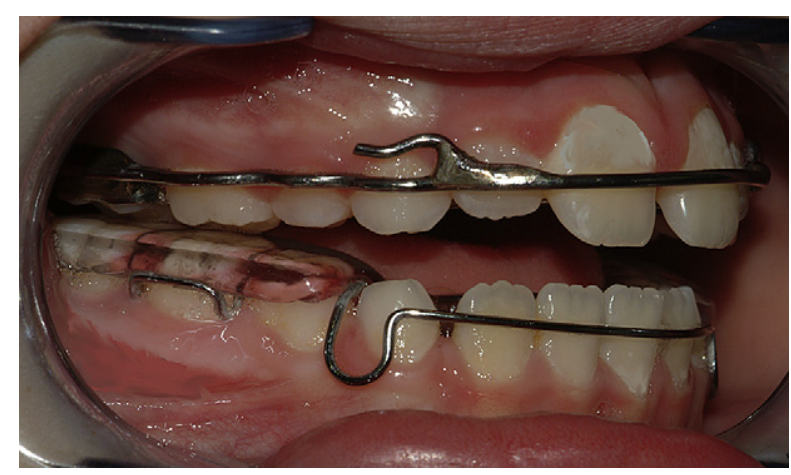

Fig 3. The same boy (age, 10 years 2 months) at T2: double-arch appliance with vestibular hooks in the maxillary arch and removable BB appliance in the mandibular arch.

of the 2 perpendicular lines from Points A and B to the functional occlusal plane), and maxillomandibular differential (difference between Co-A and Co-Gn); (5) vertical skeletal: SN-palatal plane (PP) angle, SN-GoMe angle, SN-GoGn angle, FH-PP angle, FH-mandibular plane (MP) angle, PP-MP angle, and gonial angle (ArGo-Me); and (7) dental measurements: maxillary central incisor (U1)-SN angle, U1-FH angle, IMPA angle, interincisal angle, OJ, overbite (OB), molar relationship, and U6-SePtm. 


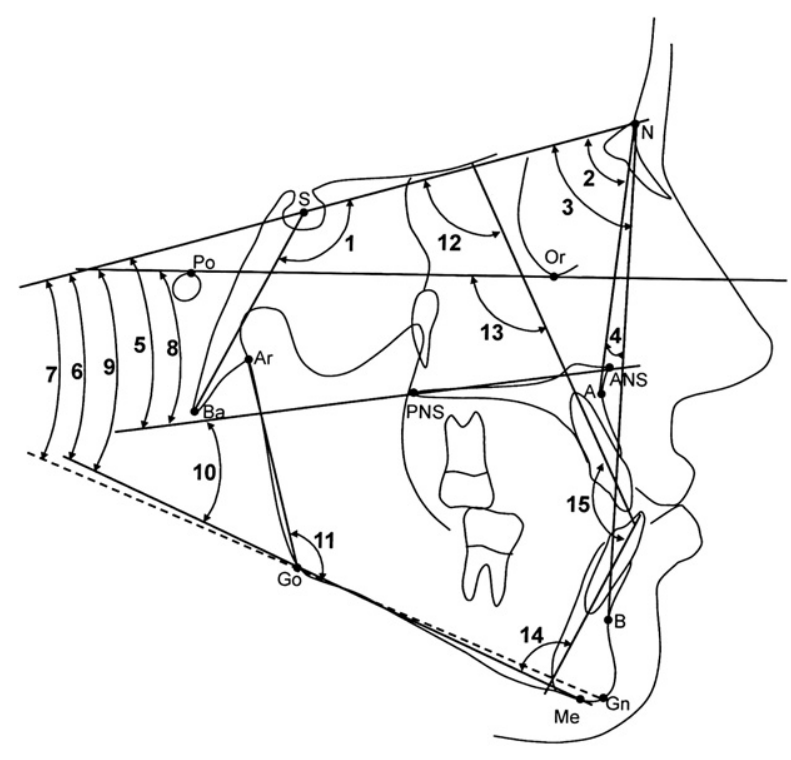

Fig 4. Cephalometric angular measurements: $1, \mathrm{NSBa}$; 2, SNA; 3, SNB; 4, ANB; 5, SN-PP; 6, SN-GoMe; 7, SN-GoGn; 8, FH-PP; 9, FH-MP; 10, PP-MP; 11, gonial angle; 12, U1-SN; 13, U1-FH; 14, IMPA; 15, interincisal angle.

\section{Statistical analysis}

Descriptive statistics were calculated for all cephalometric measures at T1, T2, and T3 for the treated and control groups. Additionally, mean differences and standard deviations were calculated for the changes from $\mathrm{T} 2$ to $\mathrm{T} 1, \mathrm{~T} 3$ to $\mathrm{T} 2$ and $\mathrm{T} 3$ to $\mathrm{T} 1$ in both groups.

The data were analyzed with statistical softwares (version 12.0, SPSS, Chicago, Ill; and SigmaStat 3.5, Systat Software, Point Richmond, Calif). Statistical significance was tested at $P<0.05, P<0.01$, and $P<0.001$.

The homogeneity between the treated and control groups (type of malocclusion, skeletal maturity at each observation time, sex distribution, and mean duration of observation intervals) allowed for comparisons without annualizing the data. Because of relatively few control subjects, the data did not have a normal distribution (Shapiro-Wilks test). Therefore, nonparametric statistics were used. The calculated power of the study, however, was adequate (0.85).

Before making the comparisons of the longitudinal changes, significant differences between the craniofacial starting forms at $\mathrm{T} 1$ were assessed with the Mann-Whitney U test between the treated and control groups. The changes in cephalometric measurements between $\mathrm{T} 1$ and $\mathrm{T} 2, \mathrm{~T} 2$ and $\mathrm{T} 3$, and $\mathrm{T} 1$ and $\mathrm{T} 3$ in the treated group were compared with the Friedman repeated measures analysis of variance (ANOVA) on

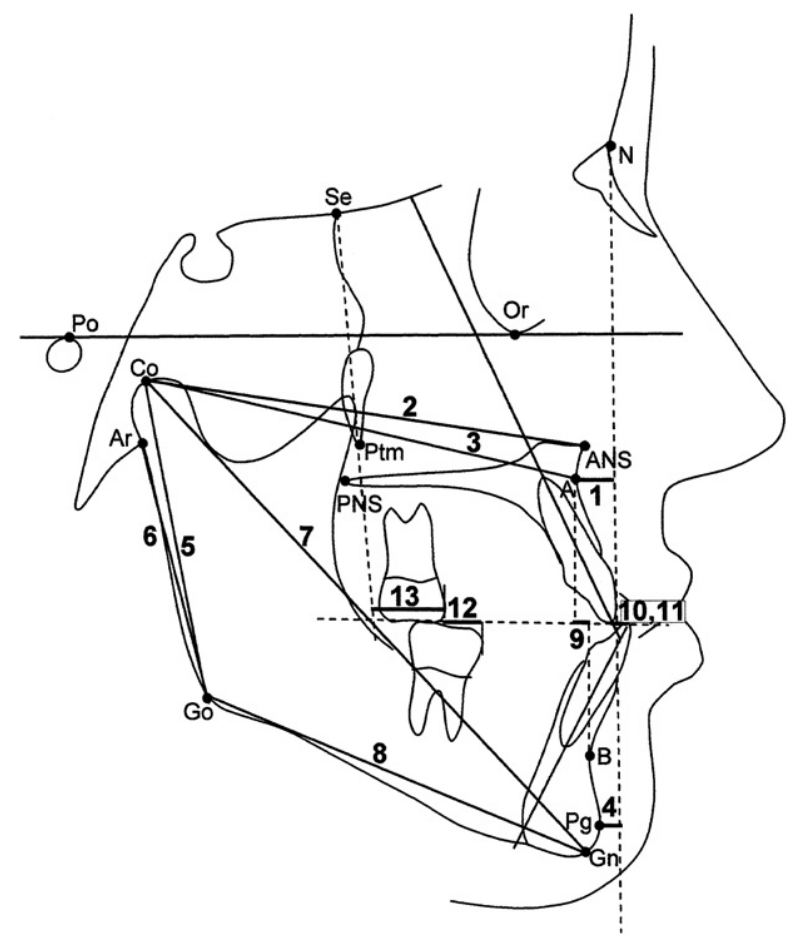

Fig 5. Cephalometric linear measurements: $1, A$ to $N$ perpendicular; 2, Co-ANS; 3, Co-A; 4, Pg to N perpendicular; 5, Co-Go; 6, Ar-Go; 7, Co-Gn; 8, Go-Gn; 9 , Wits appraisal; 10, OJ; 11, OB; 12, molar relationship; 13, U6-SePtm.

ranks with the Tukey post-hoc test $(P<0.05)$ to determine significant active treatment, posttreatment, and overall changes, respectively. To assess the differences between the treated and control groups at $\mathrm{T} 1$ to $\mathrm{T} 2$, T2 to T3, and overall T1 to T3 changes, MannWhitney $\mathrm{U}$ tests $(P<0.05, P<0.01, P<0.001)$ were used.

\section{RESULTS}

Analysis of the starting forms (Table II) showed that the treated and control groups had no statistically significant differences in craniofacial characteristics at $\mathrm{T} 1$. The only exception was a significantly greater buccal inclination of the maxillary and mandibular incisors at $\mathrm{T} 1$ in the treated group that led to a significantly smaller interincisal angle in the treated group.

Statistical analysis of the treatment and posttreatment changes in the treated group (Table III) showed several significant changes from T1 to T2. Sagittal maxillary position (SNA, A-N perpendicular), midfacial length (Co-A, Co-ANS), and mandibular total length (Co-Gn) were significantly greater at T2 than at T1. The values for the ANB angle and Wits appraisal 
Table II. Statistical comparison of cephalometric measurements between the groups at $\mathrm{T} 1$

\begin{tabular}{|c|c|c|c|c|c|}
\hline \multirow[b]{2}{*}{ Cephalometric measurement } & \multicolumn{2}{|c|}{ Treated group $(n=22)$} & \multicolumn{2}{|c|}{ Control group $(n=12)$} & \multirow[b]{2}{*}{ Mann-Whitney $U$ test } \\
\hline & Mean & $S D$ & Mean & $S D$ & \\
\hline \multicolumn{6}{|l|}{ Cranial flexure } \\
\hline $\mathrm{NSBa}\left({ }^{\circ}\right)$ & 128.4 & 5.1 & 128.0 & 5.4 & 0.4 \\
\hline \multicolumn{6}{|l|}{ Maxillary skeletal } \\
\hline SNA $\left(^{\circ}\right)$ & 80.5 & 4.3 & 77.8 & 4.0 & 1.7 \\
\hline A-N perpendicular (mm) & -0.8 & 2.7 & -1.3 & 3.3 & 0.5 \\
\hline Co-ANS (mm) & 77.6 & 5.0 & 79.0 & 3.1 & -1.4 \\
\hline Co-A (mm) & 75.2 & 5.0 & 74.6 & 2.8 & 0.6 \\
\hline \multicolumn{6}{|l|}{ Mandibular skeletal } \\
\hline $\mathrm{SNB}\left({ }^{\circ}\right)$ & 80.0 & 4.3 & 78.0 & 3.7 & 2.0 \\
\hline Pg-N perpendicular (mm) & -3.1 & 5.1 & -6.9 & 5.6 & 3.8 \\
\hline Co-Go $(\mathrm{mm})$ & 45.3 & 4.1 & 45.9 & 3.2 & -0.6 \\
\hline Ar-Go (mm) & 38.0 & 4.3 & 38.0 & 3.1 & 0.0 \\
\hline Co-Gn (mm) & 99.7 & 5.3 & 98.8 & 5.3 & 0.9 \\
\hline Go-Gn (mm) & 65.3 & 3.5 & 62.7 & 3.6 & 2.6 \\
\hline \multicolumn{6}{|l|}{ Skeletal difference } \\
\hline $\mathrm{ANB}\left({ }^{\circ}\right)$ & 0.5 & 2.0 & -0.3 & 2.4 & -1.2 \\
\hline Wits appraisal (mm) & -6.2 & 2.6 & -7.6 & 4.1 & 1.4 \\
\hline Maxillomandibular differential (mm) & 24.5 & 2.6 & 24.1 & 3.7 & 0.4 \\
\hline \multicolumn{6}{|l|}{ Vertical skeletal } \\
\hline $\mathrm{SN}-\mathrm{PP}\left({ }^{\circ}\right)$ & 7.7 & 3.2 & 10.4 & 3.3 & -2.7 \\
\hline $\mathrm{SN}-\mathrm{GoMe}\left({ }^{\circ}\right)$ & 37.4 & 6.3 & 40.9 & 5.5 & -3.5 \\
\hline SN-GoGn $\left(^{\circ}\right)$ & 35.3 & 6.2 & 38.3 & 5.7 & -3.0 \\
\hline FH-PP $\left(^{\circ}\right)$ & -0.9 & 3.5 & 1.0 & 2.4 & -1.9 \\
\hline FH-MP $\left({ }^{\circ}\right)$ & 28.8 & 5.3 & 31.6 & 3.8 & -2.8 \\
\hline PP-MP $\left(^{\circ}\right)$ & 29.6 & 6.4 & 30.5 & 4.0 & -0.9 \\
\hline Gonial angle $(\mathrm{Ar}-\mathrm{Go}-\mathrm{Me})\left({ }^{\circ}\right)$ & 134.1 & 6.3 & 135.5 & 4.4 & -1.4 \\
\hline \multicolumn{6}{|l|}{ Dental } \\
\hline $\mathrm{U} 1-\mathrm{SN}\left(^{\circ}\right)$ & 107.1 & 9.6 & 98.0 & 8.2 & $9.1^{*}$ \\
\hline $\mathrm{U} 1-\mathrm{FH}\left(^{\circ}\right)$ & 115.7 & 8.0 & 107.3 & 8.5 & $8.4 *$ \\
\hline $\operatorname{IMPA}\left({ }^{\circ}\right)$ & 88.1 & 5.5 & 80.6 & 6.4 & $7.5^{\dagger}$ \\
\hline Interincisal angle $\left(^{\circ}\right)$ & 127.5 & 9.6 & 140.5 & 13.5 & $-13.0^{\dagger}$ \\
\hline $\mathrm{OJ}(\mathrm{mm})$ & -1.2 & 2.0 & -2.1 & 2.5 & 0.9 \\
\hline $\mathrm{OB}(\mathrm{mm})$ & 0.3 & 1.1 & -1.3 & 2.4 & 1.6 \\
\hline Molar relationship (mm) & 3.0 & 1.1 & 4.0 & 3.0 & -1.0 \\
\hline U6-SePtm (mm) & 20.7 & 3.6 & 21.7 & 2.9 & -1.0 \\
\hline
\end{tabular}

$* P<0.05 ;{ }^{\dagger} P<0.01$.

showed significant improvements. The inclination of the PP to the cranial base or to the FH plane (SN-PP, FH-PP) showed significantly smaller values at T2; PPMP, on the contrary, had significantly greater values at T2. The same occurred for OJ. A positive OJ was obtained in all subjects at T2 (range, 0.3-4.1 mm). Molar relationship had a significantly smaller value at $\mathrm{T} 2$ than at T1, whereas U6-SePtm showed a significant increase. There were no significant changes in the axial inclination of the maxillary and mandibular teeth in the treatment group from $\mathrm{T} 1$ to $\mathrm{T} 2$.

Some cephalometric variables showed significant changes from T2 to T3 in the treated group. Maxillary skeletal cephalometric measurements were stable from T2 to T3. Mandibular body (Go-Gn) and ramus length (Co-Go) were significantly greater at $\mathrm{T} 3$ than at $\mathrm{T} 2$.
There were significant rebounds in the ANB angle and maxillomandibular differential, in 2 vertical dimensions (FH-PP, PP-MP), and in molar relationship at T3. At T3, only 1 subject had an end-to-end incisal relationship, whereas all others had a positive OJ (range, 0.4-4.7 mm).

The statistical comparison of the overall changes from $\mathrm{T} 1$ to $\mathrm{T} 3$ showed significantly greater increases in maxillary sagittal position (SNA) and midfacial lenght (Co-A and Co-ANS). At T2, there were significantly greater values than at $\mathrm{T} 1$ for total mandibular length (Co-Gn), mandibular body (Go-Gn), and ramus length (Ar-Go and Co-Go); the same occurred for ANB angle, Wits appraisal, and maxillomandibular differential. No vertical skeletal cephalometric measurements showed significant changes from $\mathrm{T} 1$ to $\mathrm{T} 3$. 
Table III. Treatment and posttreatment changes in the treated group

\begin{tabular}{|c|c|c|c|c|c|c|c|c|c|}
\hline \multirow[b]{2}{*}{ Cephalometric measurement } & \multicolumn{2}{|c|}{ Treated group $T 1$} & \multicolumn{2}{|c|}{ Treated group $T 2$} & \multicolumn{2}{|c|}{ Treated group $T 3$} & \multicolumn{3}{|c|}{$\begin{array}{c}\text { Statistical comparisons } \\
\text { (Friedman repeated measures } \\
\text { ANOVA on ranks) }\end{array}$} \\
\hline & Mean & $S D$ & Mean & $S D$ & Mean & $S D$ & $T 2-T 1$ & $T 3-T 2$ & $T 3-T 1$ \\
\hline \multicolumn{10}{|l|}{ Cranial flexure } \\
\hline $\operatorname{NSBa}\left({ }^{\circ}\right)$ & 128.4 & 5.1 & 127.8 & 4.2 & 128.4 & 5.8 & -0.6 & 0.6 & 0.0 \\
\hline \multicolumn{10}{|l|}{ Maxillary skeletal } \\
\hline SNA $\left(^{\circ}\right)$ & 80.5 & 4.3 & 82.6 & 4.1 & 82.4 & 4.9 & $2.1^{*}$ & -0.2 & $1.9 *$ \\
\hline A-N perpendicular (mm) & -0.8 & 2.7 & 0.4 & 2.3 & 0.1 & 3.1 & $1.2^{*}$ & -0.3 & 0.9 \\
\hline Co-ANS (mm) & 77.6 & 5.0 & 82.3 & 6.0 & 84.3 & 6.5 & $4.7^{*}$ & 2.0 & $6.7 *$ \\
\hline Co-A (mm) & 75.2 & 5.0 & 80.0 & 5.9 & 81.4 & 6.4 & $4.8^{*}$ & 1.4 & $6.2 *$ \\
\hline \multicolumn{10}{|l|}{ Mandibular skeletal } \\
\hline $\mathrm{SNB}\left({ }^{\circ}\right)$ & 80.0 & 4.3 & 79.9 & 3.8 & 80.7 & 4.9 & -0.1 & 0.8 & 0.7 \\
\hline Pg-N perpendicular $(\mathrm{mm})$ & -3.1 & 5.1 & -4.4 & 6.1 & -2.1 & 7.1 & -1.3 & 2.3 & 1.0 \\
\hline Co-Go (mm) & 45.3 & 4.1 & 47.4 & 5.3 & 49.8 & 5.6 & 2.1 & $2.4^{*}$ & $4.5^{*}$ \\
\hline Ar-Go (mm) & 38.0 & 4.3 & 39.7 & 5.1 & 41.9 & 5.5 & 1.7 & 2.2 & $3.9 *$ \\
\hline $\mathrm{Co}-\mathrm{Gn}(\mathrm{mm})$ & 99.7 & 5.3 & 104.3 & 5.9 & 108.7 & 7.5 & $4.6^{*}$ & 4.4 & $9.0 *$ \\
\hline Go-Gn (mm) & 65.3 & 3.5 & 68.0 & 3.8 & 71.1 & 4.3 & 2.7 & $3.1^{*}$ & $5.8^{*}$ \\
\hline \multicolumn{10}{|l|}{ Skeletal difference } \\
\hline $\operatorname{ANB}\left({ }^{\circ}\right)$ & 0.5 & 2.0 & 2.7 & 2.5 & 1.7 & 2.6 & $2.2^{*}$ & $-1.0 *$ & $1.2 *$ \\
\hline Wits appraisal (mm) & -6.2 & 2.6 & -4.4 & 2.1 & -4.1 & 2.7 & $1.8^{*}$ & 0.3 & $2.1^{*}$ \\
\hline Maxillomandibular differential (mm) & 24.5 & 2.6 & 24.3 & 3.7 & 27.3 & 4.9 & -0.2 & $3.0^{*}$ & $2.8^{*}$ \\
\hline \multicolumn{10}{|l|}{ Vertical skeletal } \\
\hline SN-PP $\left({ }^{\circ}\right)$ & 7.7 & 3.2 & 5.8 & 3.0 & 6.7 & 4.1 & $-1.9^{*}$ & 0.9 & -1.0 \\
\hline $\mathrm{SN}-\mathrm{GoMe}\left({ }^{\circ}\right)$ & 37.4 & 6.3 & 37.6 & 7.0 & 36.9 & 7.1 & 0.2 & -0.7 & -0.5 \\
\hline SN-GoGn $\left(^{\circ}\right)$ & 35.3 & 6.2 & 35.7 & 7.0 & 34.7 & 6.9 & 0.4 & -1.0 & -0.6 \\
\hline FH-PP $\left(^{\circ}\right)$ & -0.9 & 3.5 & -2.1 & 3.2 & -1.2 & 3.7 & $-1.2 *$ & $0.9^{*}$ & -0.3 \\
\hline FH-MP $\left(^{\circ}\right)$ & 28.8 & 5.3 & 29.7 & 6.4 & 29.1 & 6.3 & 0.9 & -0.6 & 0.3 \\
\hline PP-MP $\left({ }^{\circ}\right)$ & 29.6 & 6.4 & 31.8 & 6.7 & 30.2 & 6.5 & $2.2^{*}$ & $-1.6^{*}$ & 0.6 \\
\hline Gonial angle $(\mathrm{Ar}-\mathrm{Go}-\mathrm{Me})\left(^{\circ}\right)$ & 134.1 & 6.3 & 134.2 & 5.5 & 133.3 & 5.2 & 0.1 & -0.9 & -0.8 \\
\hline \multicolumn{10}{|l|}{ Dental } \\
\hline $\mathrm{U} 1-\mathrm{SN}\left({ }^{\circ}\right)$ & 107.1 & 9.6 & 107.1 & 7.6 & 109.6 & 7.7 & 0.0 & 2.5 & 2.5 \\
\hline $\mathrm{U} 1-\mathrm{FH}\left(^{\circ}\right)$ & 115.7 & 8.0 & 115.0 & 6.6 & 117.4 & 6.8 & -0.7 & 2.4 & 1.7 \\
\hline $\operatorname{IMPA}\left({ }^{\circ}\right)$ & 88.1 & 5.5 & 87.7 & 6.3 & 86.7 & 6.1 & -0.4 & -1.0 & -1.4 \\
\hline Interincisal angle $\left({ }^{\circ}\right)$ & 127.5 & 9.6 & 127.6 & 7.1 & 126.8 & 8.1 & 0.1 & -0.8 & -0.7 \\
\hline $\mathrm{OJ}(\mathrm{mm})$ & -1.2 & 2.0 & 1.9 & 1.0 & 1.6 & 1.0 & $3.1^{*}$ & -0.3 & $2.8^{*}$ \\
\hline $\mathrm{OB}(\mathrm{mm})$ & 0.3 & 1.1 & 1.0 & 2.2 & 1.5 & 1.5 & 0.7 & 0.5 & $1.2 *$ \\
\hline Molar relationship (mm) & 3.0 & 1.1 & 0.0 & 1.7 & 1.9 & 1.5 & $-3.0^{*}$ & $1.9^{*}$ & -1.1 \\
\hline U6-SePtm (mm) & 20.7 & 3.6 & 24.8 & 3.1 & 26.2 & 3.8 & $4.1^{*}$ & 1.4 & $5.5^{*}$ \\
\hline
\end{tabular}

$* P<0.05$.

Significantly greater increases were found for OJ, OB, and U6-SePtm.

The statistical comparisons of the changes between $\mathrm{T} 1$ and $\mathrm{T} 2$ in the treated and control groups (Table IV) showed many significant active treatment effects produced by the FM-BB protocol. All maxillary skeletal measures showed significant improvements in the treated group vs the controls. In particular, maxillary length (Co-A) had an average improvement in the treated subjects over the controls of $2.8 \mathrm{~mm}$. No significant effects of therapy were found for the mandibular skeletal variables. Intermaxillary skeletal variables showed significant improvements, with an average increase in ANB of $2.8^{\circ}$, an average increase in the Wits appraisal of $2.5 \mathrm{~mm}$, and an average reduction in the maxillomandibular differential of $-4.3 \mathrm{~mm}$. A significant counterclockwise rotation of the PP was found in the treated group, which led to a significant increase in intermaxillary divergence (PP-MP) during treatment. No significant clockwise rotation of the mandible was found. OJ improved significantly between $\mathrm{T} 1$ and $\mathrm{T} 2$ in the treatment group vs the controls $(2.6 \mathrm{~mm})$, and the molar relationship also improved $(-4.7 \mathrm{~mm})$. The maxillary molars were moved anteriorly significantly by active treatment $(2.7 \mathrm{~mm}$ more than the controls). No significant differences in posttreatment changes (T2-T3) were found between the treated and control groups (Table IV). The only exception was a significant closure of the gonial angle in the treated group $\left(-2.7^{\circ}\right.$ over the controls). 
Table IV. Statistical comparisons of the changes between groups

\begin{tabular}{|c|c|c|c|c|c|c|c|c|c|c|c|c|c|c|c|}
\hline \multirow[b]{2}{*}{ Cephalometric measurement } & \multicolumn{2}{|c|}{$\begin{array}{l}\text { Treated } \\
\text { group } \\
\text { (TG) T2-T1 }\end{array}$} & \multicolumn{2}{|c|}{$\begin{array}{l}\text { Treated } \\
\text { group } \\
(T G) T 3-T 2\end{array}$} & \multicolumn{2}{|c|}{$\begin{array}{l}\text { Treated } \\
\text { group } \\
(T G) T 3-T 1\end{array}$} & \multicolumn{2}{|c|}{$\begin{array}{l}\text { Control } \\
\text { group } \\
\text { (CG) T2-T1 }\end{array}$} & \multicolumn{2}{|c|}{$\begin{array}{l}\text { Control } \\
\text { group } \\
\text { (CG) T3-T2 }\end{array}$} & \multicolumn{2}{|c|}{$\begin{array}{l}\text { Control } \\
\text { group } \\
\text { (CG) T3-T1 }\end{array}$} & \multicolumn{3}{|c|}{$\begin{array}{c}\text { Statistical } \\
\text { comparisons } \\
\text { (Mann-Whitney U test) }\end{array}$} \\
\hline & Mean & $S D$ & Mean & $S D$ & Mean & $S D$ & Mean & $S D$ & Mean & $S D$ & Mean & $S D$ & $\begin{array}{c}T G \text { vs } C G \\
T 2-T 1\end{array}$ & $\begin{array}{l}T G \text { vs } C G \\
\quad T 3-T 2\end{array}$ & $\begin{array}{c}T G \text { vs } C G \\
\quad T 3-T 1\end{array}$ \\
\hline \multicolumn{16}{|l|}{ Cranial flexure } \\
\hline $\mathrm{NSBa}\left({ }^{\circ}\right)$ & -0.6 & 3.1 & 0.6 & 2.8 & 0.0 & 2.3 & 0.5 & 4.4 & -1.8 & 3.7 & -1.2 & 3.1 & -1.1 & 2.4 & 1.2 \\
\hline \multicolumn{16}{|l|}{ Maxillary skeletal } \\
\hline SNA $\left({ }^{\circ}\right)$ & 2.1 & 2.8 & -0.2 & 1.8 & 1.9 & 3.1 & -0.6 & 1.2 & 0.1 & 1.4 & -0.5 & 1.4 & $2.7^{\ddagger}$ & -0.3 & $2.4^{\dagger}$ \\
\hline A-N perpendicular (mm) & 1.3 & 3.0 & -0.3 & 2.1 & 1.0 & 3.0 & -1.2 & 2.0 & 0.0 & 1.9 & -1.1 & 2.1 & $2.5^{\dagger}$ & -0.3 & $2.1^{*}$ \\
\hline Co-ANS (mm) & 4.7 & 3.1 & 2.0 & 4.6 & 6.7 & 5.6 & 2.1 & 2.8 & 3.3 & 4.4 & 5.5 & 4.5 & 2.6 & -1.3 & 1.2 \\
\hline Co-A (mm) & 4.9 & 3.1 & 1.4 & 4.3 & 6.3 & 5.4 & 2.1 & 2.7 & 2.7 & 3.5 & 4.8 & 4.1 & $2.8^{*}$ & -1.3 & 1.5 \\
\hline \multicolumn{16}{|l|}{ Mandibular skeletal } \\
\hline $\mathrm{SNB}\left({ }^{\circ}\right)$ & -0.1 & 2.6 & 0.8 & 1.7 & 0.7 & 2.6 & 0.0 & 1.4 & 0.6 & 1.8 & 0.6 & 1.6 & -0.1 & 0.2 & 0.1 \\
\hline Pg-N perpendicular $(\mathrm{mm})$ & -1.3 & 6.1 & 2.2 & 4.8 & 0.9 & 5.0 & 0.2 & 4.3 & 2.7 & 3.0 & 2.9 & 5.1 & -1.5 & -0.5 & -2.0 \\
\hline Co-Go (mm) & 2.1 & 3.3 & 2.4 & 4.3 & 4.5 & 5.3 & 3.5 & 3.3 & 2.8 & 5.0 & 6.3 & 4.2 & -1.4 & -0.4 & -1.8 \\
\hline Ar-Go (mm) & 1.7 & 3.1 & 2.2 & 3.6 & 3.9 & 4.6 & 2.7 & 3.5 & 2.6 & 4.4 & 5.3 & 3.8 & -1.0 & -0.4 & -1.4 \\
\hline Co-Gn (mm) & 4.6 & 3.8 & 4.4 & 5.6 & 9.0 & 7.1 & 6.2 & 5.2 & 5.9 & 7.0 & 12.2 & 6.7 & -1.6 & -1.5 & -3.2 \\
\hline Go-Gn (mm) & 2.6 & 3.9 & 3.1 & 2.9 & 5.7 & 4.0 & 3.8 & 3.7 & 2.9 & 3.7 & 6.7 & 5.0 & -1.2 & 0.2 & -1.0 \\
\hline \multicolumn{16}{|l|}{ Skeletal difference } \\
\hline $\operatorname{ANB}\left({ }^{\circ}\right)$ & 2.2 & 1.6 & -1.0 & 1.6 & 1.2 & 1.9 & -0.6 & 1.2 & -0.5 & 2.0 & -1.1 & 2.0 & $2.8^{\ddagger}$ & -0.5 & $2.3^{*}$ \\
\hline Wits appraisal $(\mathrm{mm})$ & 1.8 & 3.3 & 0.3 & 3.1 & 2.1 & 3.0 & -0.7 & 3.8 & 0.5 & 2.1 & -0.2 & 4.0 & $2.5^{*}$ & -0.2 & 2.3 \\
\hline $\begin{array}{l}\text { Maxillomandibular } \\
\text { differential }(\mathrm{mm})\end{array}$ & -0.2 & 2.3 & 3.0 & 2.4 & 2.8 & 3.0 & 4.1 & 3.5 & 3.2 & 4.5 & 7.4 & 4.4 & $-4.3^{\ddagger}$ & -0.2 & $-4.6^{\ddagger}$ \\
\hline \multicolumn{16}{|l|}{ Vertical skeletal } \\
\hline SN-PP $\left(^{\circ}\right)$ & -1.9 & 2.5 & 0.9 & 2.4 & -1.0 & 2.4 & -0.1 & 3.3 & -0.3 & 2.8 & -0.4 & 3.6 & $-1.8^{*}$ & 1.2 & -0.6 \\
\hline SN-GoMe $\left(^{\circ}\right)$ & 0.2 & 2.4 & -0.6 & 2.1 & -0.5 & 2.4 & -0.4 & 3.7 & -0.1 & 2.6 & -0.5 & 4.5 & 0.6 & -0.5 & 0.0 \\
\hline SN-GoGn $\left({ }^{\circ}\right)$ & 0.4 & 2.3 & -1.0 & 1.6 & -0.6 & 2.4 & -0.3 & 3.6 & -1.0 & 2.7 & -1.3 & 4.8 & 0.7 & 0.0 & 0.7 \\
\hline FH-PP $\left(^{\circ}\right)$ & -1.2 & 3.4 & 0.9 & 2.4 & -0.3 & 3.2 & 0.7 & 2.6 & 0.3 & 2.5 & 1.0 & 2.8 & $-1.9 *$ & 0.6 & -1.3 \\
\hline FH-MP $\left(^{\circ}\right)$ & 0.9 & 3.3 & -0.6 & 2.6 & 0.3 & 3.2 & 0.4 & 2.6 & 0.4 & 1.9 & 0.8 & 3.4 & 0.5 & -1.0 & -0.5 \\
\hline PP-MP $\left({ }^{\circ}\right)$ & 2.1 & 2.2 & -1.5 & 2.1 & 0.6 & 2.6 & -0.3 & 1.6 & 0.2 & 3.0 & -0.1 & 3.3 & $2.4^{\dagger}$ & -1.7 & 0.7 \\
\hline $\begin{array}{l}\text { Gonial angle } \\
\left(\text { Ar-Go-Me) }\left({ }^{\circ}\right)\right.\end{array}$ & 0.1 & 3.3 & -0.9 & 2.6 & -0.8 & 3.5 & -0.8 & 3.0 & 1.8 & 1.8 & 1.0 & 3.1 & 0.9 & $-2.7 \dagger$ & -1.8 \\
\hline \multicolumn{16}{|l|}{ Dental } \\
\hline $\mathrm{U} 1-\mathrm{SN}\left({ }^{\circ}\right)$ & 0.1 & 5.5 & 2.4 & 3.9 & 2.5 & 5.7 & 1.6 & 6.5 & 2.2 & 7.2 & 3.8 & 7.7 & -1.5 & 0.2 & -1.3 \\
\hline $\mathrm{U} 1-\mathrm{FH}\left(^{\circ}\right)$ & -0.7 & 5.1 & 2.4 & 4.7 & 1.7 & 5.7 & 0.8 & 6.7 & 1.7 & 7.3 & 2.5 & 7.4 & -1.5 & 0.7 & -0.8 \\
\hline $\operatorname{IMPA}\left({ }^{\circ}\right)$ & -0.4 & 6.2 & -0.9 & 5.4 & -1.3 & 5.4 & 0.7 & 4.7 & -2.9 & 3.7 & -2.2 & 6.1 & -1.1 & 2.0 & 0.9 \\
\hline Interincisal angle $\left({ }^{\circ}\right)$ & 0.1 & 7.5 & -0.8 & 6.6 & -0.7 & 7.4 & -1.9 & 8.5 & 0.8 & 9.0 & -1.1 & 9.6 & 2.0 & -1.6 & 0.4 \\
\hline $\mathrm{OJ}(\mathrm{mm})$ & 3.1 & 2.1 & -0.3 & 1.0 & 2.8 & 2.4 & 0.5 & 1.7 & -0.2 & 1.5 & 0.3 & 2.0 & $2.6^{\ddagger}$ & -0.1 & $2.5^{\dagger}$ \\
\hline $\mathrm{OB}(\mathrm{mm})$ & 0.7 & 1.8 & 0.5 & 1.7 & 1.3 & 1.2 & 1.0 & 2.2 & -0.4 & 1.1 & 0.6 & 2.0 & -0.3 & 0.9 & 0.7 \\
\hline Molar relationship (mm) & -3.0 & 1.8 & 1.9 & 1.5 & -1.1 & 1.6 & 1.7 & 1.2 & 0.6 & 4.1 & 2.3 & 4.4 & $-4.7^{\ddagger}$ & 1.3 & $-3.4^{\dagger}$ \\
\hline U6-SePtm (mm) & 4.1 & 4.0 & 1.3 & 3.1 & 5.4 & 3.0 & 1.4 & 3.3 & 1.0 & 4.5 & 2.4 & 5.8 & $2.7 *$ & 0.3 & $3.0^{*}$ \\
\hline
\end{tabular}

${ }^{*} P<0.05 ;{ }^{\dagger} P<0.01 ;{ }^{\ddagger} P<0.001$.

The evaluation of the overall treatment and posttreatment changes (T3-T1) between the 2 groups (Fig 6) showed several significant effects of active therapy with the FM-BB protocol followed by a posttreatment period without retention appliances. Significant improvements in the treated group were found for SNA angle $\left(2.4^{\circ}\right)$, Point $\mathrm{A}$ to $\mathrm{N}$ perpendicular $(2.1 \mathrm{~mm})$, ANB angle $\left(2.3^{\circ}\right)$, and maxillomandibular differential $(-4.6 \mathrm{~mm})$. OJ, molar relationship, and U6-SePtm also showed significant overall improvements in the treated group compared with the untreated subjects $(2.5,-3.4$, and $3 \mathrm{~mm}$, respectively).

\section{DISCUSSION}

We analyzed the effects of a combined protocol of FM and a removable BB appliance in the mandibular arch in subjects with dentoskeletal Class III malocclusion. Specific features of the study were the following.

1. Class III subjects were treated consecutively, and they were included in the study regardless of treatment outcomes.

2. A longitudinal sample of Class III subjects with untreated Class III malocclusion served as the control group at all evaluation times. The limited number of 

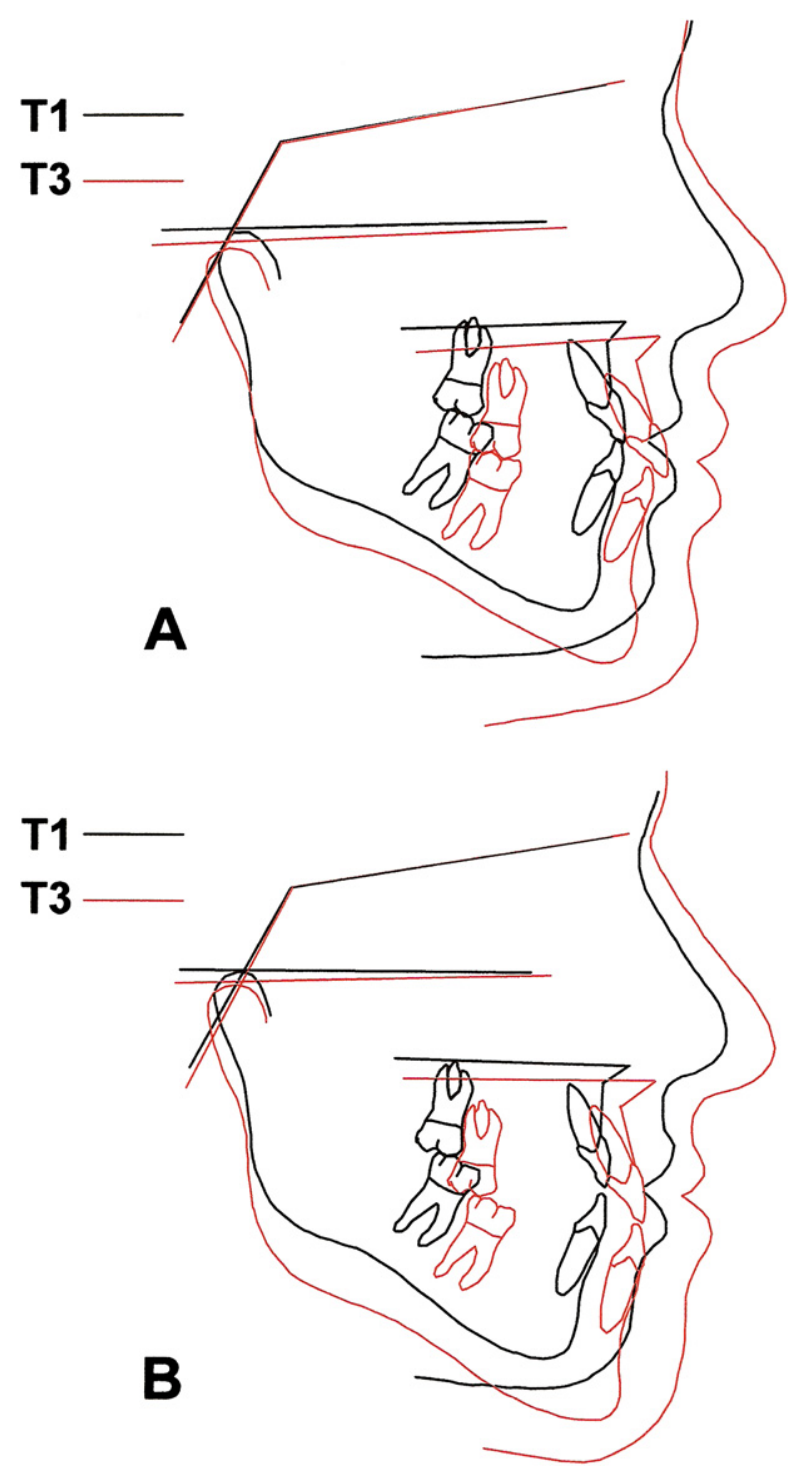

Fig 6. Craniofacial changes derived from superimpositions for the T1-T3 interval: A, treated group; B, control group.

subjects examined was because of using longitudinal records of untreated white Class III subjects that covered the circumpubertal growth period.

3. We examined both active treatment changes and posttreatment modifications. No appliance was used during the posttreatment interval. Therefore, the amount of possible posttreatment relapse tendency investigated here must be considered the maximum amount of rebound to be expected after treatment and before fixed appliance therapy to refine the occlusion.

4. All subjects were prepubertal at T1 and postpubertal at T3. The adolescent growth spurt was included in the overall observation period for all subjects in both groups.

5. The active treatment protocol consisted of a FM on a maxillary double-arch fixed appliance combined with $\mathrm{a} \mathrm{BB}$ in the mandibular arch. The main purpose of the BB was to counteract the possible tendency toward posterior rotation of the mandible; this has been shown to be an unfavorable skeletal change in both Class III subjects treated with FM and untreated Class III subjects. ${ }^{22,23}$

Although the changes in the treated group (Table III) reflect both treatment-induced and growth-induced modifications, the comparisons between treated and control groups (Table IV) refer more directly to the effectiveness of therapy vs physiologic growth trends in Class III malocclusion. Thus, the discussion of the results will focus primarily on the comparison between treated and untreated Class III subjects in both the shortterm and posttreatment observation intervals. As for comparisons with previous findings of the literature, only studies that reported the effectiveness of FM treatment without palatal expansion and that included untreated Class III controls were considered. ${ }^{5,22,23,39-41}$ Only the study by Chong et $\mathrm{al}^{22}$ included both treatment and posttreatment observations. The clinical investigation by Westwood et al, ${ }^{15}$ who described the effectiveness of maxillary expansion and protraction therapy in Class III malocclusion at a postpubertal observation, will be included in the discussion as well.

\section{Sagittal maxillomandibular changes}

The response of the craniofacial complex to active orthopedic treatment of Class III malocclusion with the FM-BB protocol consisted of significant changes in maxillary growth and position in the T1-T2 shortterm period. Both angular and linear sagittal measurements of the maxilla showed significant improvements during active treatment between 2.5 and 2.8 millimeters or degrees, whereas no significant effect of FM-BB therapy was found in the mandible. However, although not significant, the changes in the mandibular variables vs the controls were generally favorable, with improvements in sagittal position or growth of the mandible from $0.1^{\circ}$ (SNB) to $1.6 \mathrm{~mm}$ (Co-Gn). These effects allowed for a highly significant improvement in the maxillomandibular skeletal relationships. ANB improved by $2.8^{\circ}$, Wits appraisal by $2.5 \mathrm{~mm}$, and maxillomandibular differential by a notable 4.3 mm over the controls.

The results that refer to the favorable maxillary effect of treatment in the short term agree with most of the literature on FM therapy. ${ }^{15,23,39-41}$ On the 
contrary, Chong et $\mathrm{al}^{22}$ did not report significant improvement in maxillary position after FM therapy, in either the short or the long term. The amount of forward displacement of the maxilla during active treatment was relatively greater in our study compared with previous reports; this could be ascribed to the use of the splints in the mandibular dentition that facilitated the forward movement of the maxillary arch or the prolonged time of FM wear (12-18 months on average) in this study compared with other clinical trials (usually about 7-12 months).

In the posttreatment period (T2-T3), no significant changes in the treated subjects over the controls were found. Therefore, even with no retention protocol, no actual relapse in the maxillomandibular variables was found after active treatment. On the other hand, the lack of difference between the treated and control Class III subjects in the posttreatment period confirms previous observations that, after orthopedic Class III treatment, there is a tendency for the original Class III growth pattern to reassert itself. ${ }^{22,23}$ The short posttreatment observation period should be considered when discussing the changes after active treatment.

The overall $\mathrm{T} 1$ to $\mathrm{T} 3$ changes reflected the $\mathrm{T} 1$ to $\mathrm{T} 2$ changes: a significant amount of maxillary advancement ( $2.4^{\circ}$ for the SNA angle) and significant improvements in both the ANB angle $\left(2.3^{\circ}\right)$ and the maxillomandibular differential $(4.6 \mathrm{~mm})$ in treated vs control subjects. Even though it was not statistically significant, the amount of overall reduction in mandibular growth $(-3.2 \mathrm{~mm}$ for Co-Gn in treated subjects vs controls) should be mentioned as a clinically important outcome. Westwood et al ${ }^{15}$ found net differences between treated and control groups during the T1-T3 interval of about $1.5 \mathrm{~mm}$ for maxillary position and $2.5 \mathrm{~mm}$ for mandibular length, thus leading to improvement in the maxillomandibular differential $(4.1 \mathrm{~mm})$ that was similar to our study. The mandibular effects in the study by Chong et $\mathrm{al}^{22}$ agree with our findings; those authors reported a net improvement of $3.6 \mathrm{~mm}$ for mandibular length in the treated group vs the controls.

\section{Vertical changes}

Although the BB appliance and the FM were effective in preventing clockwise rotation of the mandible, orthopedic treatment induced counterclockwise rotation of the PP (about $2^{\circ}$ ) that was reflected by a similar increase in the relationship between the PP and the MP. However, this short-term change that had been described previously by Deguchi et $\mathrm{al}^{23}$ disappeared at the long-term observation. The positive effect of the $\mathrm{BB}$ in inducing anterior rotation of the mandible was mostly discernible during the posttreatment period. Although untreated Class III patients exhibited a skeletal open-bite tendency during the growth spurt, as described before, the treated group showed progressive reductions in the inclination of the MP $t o$ the FH (FH-MP, $-1^{\circ}$ ) and the PP (PP-MP, $\left.-1.7^{\circ}\right) .^{24}$ These outcomes were sustained by significant closure of the gonial angle (Ar-Go-Me, $-2.7^{\circ}$ ) in treated subjects vs controls (anterior morphogentic rotation of the mandible); this is a favorable mechanism for the stability of Class III treatment outcomes. ${ }^{4,15,24}$ After the use of a FM without the BB appliance, Deguchi et $\mathrm{al}^{23}$ reported significant posterior rotations of the mandible during and after orthopedic treatment of Class III malocclusion.

\section{Dental changes}

The effect of maxillary protraction did not cause significant protraction of the maxillary incisors or retraction of the mandibular incisors, either during therapy or at the postpubertal observation. The BB appliance with the acrylic contacting the lingual surfaces of the mandibular incisors probably played a favorable role in preventing retraction of the mandibular incisors. On the contrary, significant retroclination of the mandibular incisors was reported by Chong et al, ${ }^{22}$ Deguchi et al, ${ }^{23}$ and Westwood et al. ${ }^{15}$ Both OJ and molar relationship improved significantly (at both statistical and clinical levels) between $\mathrm{T} 1$ and $\mathrm{T} 2$, and also between $\mathrm{T} 1$ and T3. Net postpubertal improvements of $2.5 \mathrm{~mm}$ in $\mathrm{OJ}$ and about $3.5 \mathrm{~mm}$ in molar relationship were found in the treated group compared with the untreated controls. Interestingly, much of this favorable change in molar relationship could be ascribed to significant forward movement $(3 \mathrm{~mm})$ of the maxillary molars that bore the bands of the double-arch intraoral appliance for maxillary protraction. The amount of OJ correction was similar in the study by Chong et $\mathrm{al}^{22}(2.2 \mathrm{~mm})$, who evaluated treatment and posttreatment changes of FM therapy.

\section{CONCLUSIONS}

We compared the treatment effects of a FM-BB protocol in Class III malocclusion and growth changes in untreated Class III subjects. The following treatment and posttreatment craniofacial modifications were seen during an average observation period of 4 years 3 months in the circumpubertal stages of skeletal development.

1. Treatment with the FM-BB protocol induced significant dentoskeletal responses in terms of 
improvement of SNA angle, ANB angle, OJ, and molar relationship; these changes remained stable during the posttreatment period.

2. No modifications were found in vertical skeletal relationships. The BB in the mandibular arch enabled effective control of mandibular rotation and facilitated progressive closure of the gonial angle.

3. As for the dental changes associated with orthopedic treatment, the FM-BB protocol caused no proclination of the maxillary incisors or retroclination of the mandibular incisors in the long term. The significant improvement in OJ $(2.5 \mathrm{~mm})$ was entirely related to the amount of skeletal maxillary advancement.

\section{REFERENCES}

1. Delaire J. Le syndrome prognathique mandibulaire. Orthod Fr 1976;47:203-19.

2. Fränkel R. Maxillary retrusion in Class III and treatment with the function corrector III. Trans Eur Orthod Soc 1970;46: 249-59.

3. Garattini G, Levrini L, Crozzoli P, Levrini A. Skeletal and dental modifications produced by the bionator III appliance. Am J Orthod Dentofacial Orthop 1998;114:40-4.

4. Tollaro I, Baccetti T, Franchi L. Mandibular skeletal changes induced by early functional treatment of Class III malocclusion: a superimposition study. Am J Orthod Dentofacial Orthop 1995; 108:525-32.

5. Üçem TT, Ucuncu N, Yüksel S. Comparison of double-plate appliance and facemask therapy in treating Class III malocclusions. Am J Orthod Dentofacial Orthop 2004;126:672-9.

6. Graber LW. Chin cup therapy for mandibular prognathism. Am J Orthod 1977;72:23-41.

7. Ferro A, Perillo Nucci L, Ferro F, Gallo C. Long-term stability of skeletal Class III patients treated with splints, Class III elastics, and chincup. Am J Orthod Dentofacial Orthop 2003; 123:423-34.

8. Cozzani G. Extraoral traction and Class III treatment. Am J Orthod 1981;80:638-50.

9. Rey D, Angel D, Oberti G, Baccetti T. Treatment and posttreatment effects of mandibular cervical headgear followed by fixed appliances in Class III malocclusion. Am J Orthod Dentofacial Orthop 2008;133:371-8.

10. Irie M, Nakamura S. Orthopedic approach to severe skeletal Class III malocclusion. Am J Orthod 1975;67:377-92.

11. McNamara JA Jr. An orthopedic approach to the treatment of Class III malocclusion in young patients. J Clin Orthod 1987; 21:598-608

12. Mermigos J, Full CA, Andreasen G. Protraction of the maxillofacial complex. Am J Orthod Dentofacial Orthop 1990;98: 47-55.

13. Delaire J. Maxillary development revisited: relevance to the orthopaedic treatment of Class III malocclusions. Eur J Orthod 1997;19:289-311.

14. Baccetti T, McGill JS, Franchi L, McNamara JA Jr, Tollaro I. Skeletal effects of early treatment of Class III malocclusion with maxillary expansion and face-mask therapy. Am J Orthod Dentofacial Orthop 1998;113:333-43.

15. Westwood PV, McNamara JA Jr, Baccetti T, Franchi L, Sarver DM. Long-term effects of Class III treatment with rapid maxillary expansion and facemask therapy followed by fixed appliances. Am J Orthod Dentofacial Orthop 2003;123: 306-20.

16. De Toffol L, Pavoni C, Baccetti T, Franchi L, Cozza P. Orthopedic treatment outcomes in Class III malocclusion, a systematic review. Angle Orthod 2008;78:561-73.

17. Williams MD, Sarver DM, Sadowsky PL, Bradley E. Combined rapid maxillary expansion and protraction facemask in the treatment of Class III malocclusions in growing children: a prospective long-term study. Semin Orthod 1997;3: 265-74.

18. Ngan PW, Hägg U, Yiu C, Wei SH. Treatment response and longterm dentofacial adaptations to maxillary expansion and protraction. Semin Orthod 1997;3:255-64.

19. Hägg U, Tse A, Bendeus M, Rabie AB. Long-term follow-up of early treatment with reverse headgear. Eur J Orthod 2003;25: 95-102.

20. Ghiz MA, Ngan P, Gunel E. Cephalometric variables to predict future success of early orthopedic Class III treatment. Am J Orthod Dentofacial Orthop 2005;127:301-6.

21. Wells AP, Sarver DM, Proffit WR. Long-term efficacy of reverse pull headgear therapy. Angle Orthod 2006;76:915-22.

22. Chong YH, Ive JC, Artun J. Changes following the use of protraction headgear for early correction of Class III malocclusion. Angle Orthod 1996;66:351-62.

23. Deguchi T, Kanomi R, Ashizawa Y, Rosenstein SW. Very early face mask therapy in Class III children. Angle Orthod 1999;69: 349-55.

24. Baccetti T, Franchi L, McNamara JA Jr. Treatment and posttreatment craniofacial changes after rapid maxillary expansion and facemask therapy. Am J Orthod Dentofacial Orthop 2000;118: 404-13.

25. Yoshida I, Ishii H, Yamaguchi N, Mizoguchi I. Maxillary protraction and chincap appliance treatment effects and long-term changes in skeletal Class III patients. Angle Orthod 1999;69: 543-52.

26. Kuster R, Ingervall B. The effect of treatment of skeletal open bite with two types of bite-blocks. Eur J Orthod 1992;14: 489-99.

27. McNamara JA Jr. An experimental study of increased vertical dimension in the growing face. Am J Orthod 1977;71: 382-95.

28. Altuna A, Woodside DG. Response of the midface to treatment with increased vertical occlusal forces. Angle Orthod 1985;55: 251-63.

29. Woods MG, Nanda RS. Intrusion of posterior teeth with magnets. An experiment in growing baboons. Angle Orthod 1988;58: 136-50.

30. Dellinger EL. A clinical assessment of the active vertical corrector-a nonsurgical alternative for skeletal open bite treatment. Am J Orthod 1986;89:428-36.

31. Kalra V, Burstone CJ, Nanda R. Effects of a fixed magnetic appliance on the dentofacial complex. Am J Orthod Dentofacial Orthop 1989;95:467-78.

32. Kiliaridis S, Tzakis MG, Carlsson GE. Short-term and long-term effects of chewing training on occlusal perception of thickness. Scand J Dent Res 1990;98:159-66.

33. Jacobson A. Application of the "Wits" appraisal. Am J Orthod 1976;70:179-89.

34. Baccetti T, Franchi L, McNamara JA Jr. The cervical vertebral maturation (CVM) method for the assessment of optimal treatment timing in dentofacial orthopedics. Semin Orthod 2005;11: 119-29. 
35. McNamara JA Jr. A method of cephalometric evaluation. Am J Orthod 1984;86:449-69.

36. Ricketts RM. The influence of orthodontic treatment on facial growth and development. Angle Orthod 1960;30:103-33.

37. Steiner CC. Cephalometrics for you and me. Am J Orthod 1953; 39:729-55.

38. Tweed C. Clinical orthodontics. St Louis: C.V. Mosby; 1966.
39. Kiliçoğlu H, Kirliç Y. Profile changes in patients with Class III malocclusions after Delaire mask therapy. Am J Orthod Dentofacial Orthop 1998;113:453-62.

40. Yüksel S, Üçem TT, Keykubat A. Early and late facemask therapy. Eur J Orthod 2001;23:559-68.

41. Tortop T, Keykubat A, Yüksel S. Facemask therapy with and without expansion. Am J Orthod Dentofacial Orthop 2007;132:467-74. 